\title{
Diseño de un índice pronóstico clínico para el manejo de la neumonía del adulto adquirida en la comunidad
}

\author{
Fernando Saldías $\mathbf{P}^{\mathbf{1}}$, Gonzalo Farías $\mathbf{G}^{\mathbf{a}}$, Luis Villarroel $\mathbf{D}^{\mathbf{2}}$, \\ Gonzalo Valdivia $C^{2}$, José Miguel Mardónez $U^{3}$, \\ Alejandro Díaz $\mathrm{F}^{\mathbf{1}}$. \\ Development of an instrument to \\ assess the severity of community \\ acquired pneumonia among \\ hospitalized patients
}

\begin{abstract}
Background: Community acquired pneumonia (CAP) severity assessment is crucial. Aim: To develop a practical clinical severity assessment model for stratifying immunocompetent adult patients hospitalized with CAP into different management groups. Patients and methods: During a 24 months period, 455 adult patients ( 250 male, mean age $69 \pm 19$ years old) were evaluated. All the relevant clinical information recorded and they were followed during hospital stay until discharge or death. Mortality until 30 days after admission was determined. Results: The mean hospital length of stay was $9.9 \pm 9.4$ days and $76 \%$ had an underlying disease. In hospital mortality was $7.6 \%$ and $10.1 \%$ at 30 days follow up. Admission prognostic factors associated with high mortality at 30 days follow up were: advanced age, presence of comorbidity, suspicion of aspiration, duration of symptoms $\leq 2$ days, altered mental status, absence of cough, fever and chills; low blood pressure, tachypnea, hypoxemia and multilobar radiographic pulmonary infiltrates. A clinical prognostic index derived from a logistic regression analysis including five independent variables associated with mortality (confusion, comorbidity, low systolic blood pressure, temperature $<37.5^{\circ} \mathrm{C}$ and respiratory rate $>20 / \mathrm{min}$ ), enabled patients to be stratified according to increasing risk of mortality: class 1: $0.9 \%$, class 2: $4.9 \%$, class 3: $14.2 \%$, and class 4: $35.6 \%$. Conclusion: A simple clinical severity assessment tool based on confusion, comorbidity, blood pressure, temperature and respiratory rate could be used to stratify patients with CAP into different risk class categories and management groups (Rev Méd Chile 2004; 132: 1037-46).
\end{abstract}

(Key Words: Lung diseases; Pneumonia, bacterial; Pneumonia, community acquired; Prognosis)

Recibido el 28 de noviembre, 2003. Aceptado en versión corregida el 25 de mayo, 2004.

Departamentos de Enfermedades Respiratorias', Salud Pública ${ }^{2}$ y Medicina Interna ${ }^{3}$, Pontificia Universidad Católica de Chile.

aAlumno 7º Año de Medicina, Pontificia Universidad Católica de Chile.

$\overline{\text { Correspondencia a: Dr. Femando Saldías P. Departamento de }}$ Enfermedades Respiratorias, Pontificia Universidad Católica de Chile. Marcoleta 345 - 4ํㅗ Piso. Teléfonos: 6331541-3543242.

Fax: 6335255. Santiago, Chile. E mail: fsaldias@med.puc.cl 
Lia a neumonía del adulto adquirida en la comunidad (NAC) constituye un importante problema de salud pública en el ámbito mundial ${ }^{1-3}$. Es así como, a pesar de la aparición de nuevas terapias antibióticas en los últimos años, constituye la tercera causa de muerte en nuestro país y la principal causa de muerte por enfermedades infecciosas 4 . Se ha estimado que uno de cada cinco pacientes requiere hospitalización para su manejo, con una letalidad que oscila entre $10 \mathrm{y}$ $20 \%$ para los pacientes hospitalizados en sala de cuidados generales o Unidad de Intermedio, y que asciende a alrededor de 40-50\% para aquellos admitidos a la Unidad de Cuidados Intensivos ${ }^{5,6}$.

La neumonía comunitaria del adulto tiene un espectro muy amplio de presentaciones, con un riesgo de muerte que varía dependiendo de la etiología microbiana y de factores propios de cada paciente. De este modo, en un individuo joven, sin comorbilidad y sin criterios de gravedad, sería adecuado iniciar un tratamiento antibiótico oral en el medio ambulatorio, considerando que la letalidad para este grupo es inferior a 1-2\%, llegando a requerir hospitalización sólo $5 \%$ de los casos debido a complicaciones o falla de tratamiento ${ }^{5-7}$. En el paciente senescente con patologías crónicas asociadas, sería conveniente evaluar un esquema antibiótico de amplio espectro, que nos asegure la cobertura antimicrobiana adecuada con un control clínico y de laboratorio cercano $0^{5-7}$. Sin embargo, no siempre resulta fácil evaluar la gravedad y determinar el pronóstico de un determinado paciente, y se ha comunicado que los clínicos tienden a subestimar la gravedad de la neumonía 5 , lo cual puede afectar el pronóstico y la evolución del enfermo.

De este modo, se ha planteado la necesidad de contar con índices pronósticos que nos permitan valorar el riesgo real de un paciente con NAC, con el fin de ofrecerle un tratamiento efectivo, pero que a la vez no implique un aumento exagerado en la tasa de hospitalizaciones ni en la demanda de recursos de salud, con un manejo racional de los esquemas antibióticos prescritos.

Respondiendo a esta necesidad, en los últimos años se han publicado diversos estudios que intentan describir e identificar a los pacientes con mayor riesgo de muerte y que, por lo tanto, requieren ser manejados en el hospital ${ }^{1-3,5-13}$. En guías clínicas internacionales se ha recomendado el empleo de dos índices pronósticos para evaluar la gravedad de los enfermos con NAC, siendo los más ampliamente conocidos y validados en la literatura extranjera ${ }^{14,15}$. El primero, corresponde al índice pronóstico descrito por la Sociedad Británica de Tórax $(B T S)^{2}$, que fue modificado por Neill y col $(\mathrm{mBTS})^{5}$ y, más recientemente por Lim y col (CURB-65) ${ }^{16,17}$. Este último ha propuesto el empleo de cinco variables clínicas y de laboratorio (edad $\geq 65$ años, confusión mental, presión arterial $\leq 90 / 60 \mathrm{mmHg}$, frecuencia respiratoria $\geq 30 \mathrm{resp} / \mathrm{min}$ y niveles de urea en sangre $>7 \mathrm{mmol} / \mathrm{l}$ ) para estratificar a los pacientes con NAC en tres categorías de riesgo y ayudar al clínico a decidir el lugar de manejo (ambulatorio u hospitalizado).

La segunda herramienta de discriminación de gravedad, corresponde al Indice de Gravedad de la Neumonía (PSI) desarrollado en 1997 por Fine y $\mathrm{col}^{7}$, con el propósito de identificar a aquellos pacientes que se encuentran en bajo riesgo de muerte por el episodio de NAC; esto se logra a través de un proceso de dos fases, en el que inicialmente se evalúa trece elementos de la anamnesis y examen físico y posterormente se consideran elementos de laboratorio para asignar al paciente dentro de cinco categonías de riesgo, lo que permite definir el pronóstico de los enfermos, orientar el lugar de manejo, la intensidad del estudio diagnóstico y el tratamiento antimicrobiano prescrito.

En un estudio reciente, se ha comunicado en nuestro medio que el índice pronóstico de Fine (PSI) se correlaciona con el riesgo de muerte y la tasa de complicaciones en el hospital de los pacientes adultos admitidos por NAC, lo que sugiere podría ser una herramienta útil para decidir el lugar donde ellos deben ingresar (Sala, Unidad de Cuidados Intermedios o UCI) ${ }^{18,19}$.

Sin embargo, la medición de los índices pronósticos de Fine y la BTS, requiere de múltiples exámenes de laboratorio, los cuales no siempre están disponibles en el medio ambulatorio nacional y algunos de nuestros Servicios de Urgencia, lo cual limita su aplicabilidad clínica. Además, ambos índices fueron desarrollados y validados en poblaciones extranjeras, que no necesariamente representan las características clínico-epidemiológicas de la población chilena.

El principal objetivo del presente estudio ha sido identificar las variables clínico-radiográficas 
disponibles en la atención ambulatoria que se relacionan con un peor pronóstico en términos de mortalidad en adultos inmunocompetentes hospitalizados por neumonía comunitaria, y diseñar una herramienta pronóstica que pueda ser aplicable en el medio ambulatorio nacional.

\section{PACIENTES Y MÉTODO}

En el estudio se evaluaron en forma consecutiva 455 adultos inmunocompetentes hospitalizados por un episodio de neumonía adquirida en la comunidad en el Hospital Clínico de la Pontificia Universidad Católica de Chile (hospital docente de 520 camas) entre el $1^{\circ}$ de junio de 1999 y el 31 de mayo de 2001. Todos los pacientes eran inmunocompetentes, mayores de 15 años y cumplían con los criterios diagnósticos de neumonía comunitaria propuestos por Fang y cols ${ }^{20}$ y con los criterios de hospitalización sugeridos por la Sociedad Americana de Tórax ${ }^{14}$. Se excluyó a los pacientes portadores del virus de inmunodeficiencia humana (VIH), neoplasia activa (tumores sólidos o hematológicos), neutropenia (menos de 1.000 leucocitos por $\mathrm{mm}^{3}$ ), en tratamiento quimioterápico o inmunosupresor (ciclosporina, azatioprina) en los 6 meses previos a la admisión, o tratamiento con dosis $\geq 20 \mathrm{mg} /$ día de prednisona o su equivalente durante, por lo menos, un mes dentro de los seis meses anteriores a su ingreso y pacientes moribundos, en quienes el deceso por el episodio de neumonía se considera el evento terminal previsible de su enfermedad crónica de base.

Los pacientes fueron evaluados al momento del ingreso y se registraron los siguientes antecedentes clínico-epidemiológicos: edad, sexo, lugar de procedencia (hogar o centro geriátrico), presencia de comorbilidad $^{21}$ (sólo se examinaron aquellas descritas en las guías clínicas de la BTS y ATS ${ }^{14,15}$ : insuficiencia cardiaca congestiva, enfermedad pulmonar obstructiva crónica, diabetes mellitus, enfermedad neurológica crónica (esta categoría sólo incluye pacientes con enfermedad cerebrovascular con secuela motora, enfermedad de Parkinson, demencia o enfermedad neuromuscular con compromiso funcional significativo), neoplasia, insuficiencia renal o enfermedad hepática crónica), hábito tabáquico y consumo de alcohol, uso de antibióticos previo al ingreso (cualquier dosis de antimicrobianos recibida en la última semana), sospecha de aspiración bronquial (definida como cualquier condición subyacente asociada a compromiso del sensorio, pérdida del reflejo tusígeno o alteración de la deglución), cuadro clínico de presentación (presencia de fiebre, tos, expectoración mucopurulenta o hemoptoica, disnea, dolor torácico, confusión mental o escalofríos), duración de la sintomatología antes de la admisión, antecedente de hospitalización durante los últimos 12 meses, presencia de enfermedad aguda concomitante (insuficiencia cardíaca, arritmia, isquemia miocárdica, asma bronquial o EPOC reagudizado). Se registraron los signos vitales, examen físico y mental de ingreso, y la saturación arterial de oxígeno medida con oxímetro de pulso. El compromiso cualitativo de conciencia o confusión mental fue definido por la presencia de desorientación en tiempo, espacio o personas que no corresponden a una condición basal conocida 0 coma. Además, se consignaron los hallazgos de la radiografía de tórax póstero-anterior y lateral realizada en el momento de la admisión, las cuales fueron evaluadas por un radiólogo del Departamento de Radiología, quien estaba involucrado en el estudio y desconocía el cuadro clínico de los pacientes. El patrón radiográfico fue clasificado como: a) imagen de relleno alveolar, b) patrón intersticial y c) patrón mixto. El grado de extensión del compromiso radiográfico fue descrito como: a) unilobar, b) multilobar ( $\geq 2$ lóbulos) o c) bilateral. Además se consignó la presencia de derrame pleural, absceso y cavitación.

Se registraron los exámenes de laboratorio solicitados en la admisión al hospital, los cuales se han asociado con un curso clínico complicado o mayor mortalidad intrahospitalaria6-15 (hemograma, pruebas hepáticas, proteína $\mathrm{C}$ reactiva, gases arteriales, glicemia, albúmina sérica, electrolitos plasmáticos, función renal), los cuales no fueron considerados durante el presente análisis, salvo para el cálculo de los índices pronósticos PSI y CURB-657,17.

Durante la estadía en el hospital se consignaron los tratamientos antimicrobianos y la aparición de las siguientes complicaciones: admisión a UCI, uso de ventilación mecánica, arritmias (fibrilación auricular, taquicardia paroxística supraventricular), insuficiencia cardíaca congestiva, isquemia miocárdica, accidente vascular encefálico, insuficien- 
cia renal aguda, shock séptico, empiema pleural, síndrome de distrés respiratorio del adulto, infección extrapulmonar y hemorragia digestiva. Finalmente, se consignó la letalidad en el hospital y a los 30 días de seguimiento desde la admisión.

Análisis estadístico. Los resultados son expresados como valores promedio \pm desviación estándar para las variables medidas en escala numérica y en porcentaje para las medidas en escala nominal. El análisis de los datos se realizó con los programas computacionales SPSS versión 10.0 para Windows y S-Plus 2000. En una primera etapa, se realizó un análisis bivariado considerando como variable dependiente la letalidad de los pacientes durante el seguimiento a 30 días. Las variables cualitativas fueron comparadas mediante la prueba de chi cuadrado y las variables cuantitativas mediante la prueba t de Student. Se calcularon los odds ratio e intervalos de confianza (IC) para el 95\%. Las diferencias entre las variables fueron consideradas significativas con un valor de $\mathrm{p}<0,05$.

Luego se procedió a construir una herramienta pronóstica considerando sólo aquellas variables clínico-radiográficas que resultaron significativas en el análisis univariado y que a su vez tuvieran respaldo en la literatura médica ${ }^{1-3,5-19}$, asignándoles un puntaje proporcional a sus valores de odds ratio. Finalmente, los pacientes fueron agrupados en tres categorías de riesgo de muerte. El índice pronóstico derivado del análisis univariado fue examinado en los 345 pacientes que disponían de la información clínico-radiográfica necesaria para su diseño. Sin embargo, las características clínicas, evolución y pronóstico no difirieron significativamente en el grupo de enfermos con información incompleta, quienes no fueron examinados con este índice pronóstico.

En una segunda etapa y con el fin de derivar un índice pronóstico que considerara múltiples interacciones entre variables explicatorias, conservando aquellas que fuesen significativas por sí solas, se realizó un análisis de regresión logística, para lo que se categorizó a las variables continuas en intervalos, considerando los puntos de corte que otorgaran mayor aporte al modelo y que a su vez fueran clínicamente significativos. Se realizaron modelos de regresión logística stepwise en

Tabla 1. Variables clínico-radiográficas asociadas a mortalidad en pacientes adultos inmunocompetentes hospitalizados por neumonía adquirida en la comunidad. Análisis univariado

\begin{tabular}{|lrrrrr|}
\hline Factores pronósticos & Pacientes & Fallecidos & \multicolumn{1}{c}{$p$} & Odds ratio & IC 95\% \\
\hline Edad >65 años & 292 & 38 & 0,006 & 3,0 & $1,3-7,0$ \\
Comorbilidad & 338 & 43 & 0,002 & 7,4 & $1,8-30,9$ \\
Enfermedad neurológica & 89 & 22 & $<0,001$ & 4,7 & $2,8-8,9$ \\
Enfermedad hepática crónica & 20 & 5 & 0,043 & 3,2 & $1,1-9,2$ \\
Sospecha de aspiración & 55 & 15 & $<0,001$ & 4,5 & $2,2-9,0$ \\
Duración síntomas $\leq 2$ días & 40 & 11 & 0,001 & 4,3 & $2,0-9,6$ \\
Tos & 361 & 30 & 0,005 & 0,4 & $0,2-0,8$ \\
Fiebre & 299 & 23 & 0,017 & 0,5 & $0,3-0,9$ \\
Compromiso de conciencia & 111 & 27 & $<0,001$ & 5,6 & $2,9-10,6$ \\
Escalofríos & 125 & 4 & 0,002 & 0,2 & $0,1-0,6$ \\
P sistólica <90 mmHg & 33 & 12 & $<0,001$ & 6,8 & $3,1-15,2$ \\
P diastólica <60 mmHg & 106 & 17 & 0,015 & 2,2 & $1,2-4,3$ \\
F respiratoria >20 x' & 346 & 42 & 0,027 & 7,0 & $1,1-52,3$ \\
Temperatura <37,5 ${ }^{\circ}$ C & 164 & 27 & $<0,001$ & 3,1 & $1,6-5,9$ \\
SaO 2 <90\% (FiO $:$ 0,21) & 251 & 34 & 0,015 & 2,4 & $1,2-4,8$ \\
NAC multilobar & 173 & 26 & 0,009 & 2,3 & $1,2-4,2$ \\
Ingreso a UTIM-UCI & 161 & 26 & $<0,001$ & 4,6 & $2,1-10,2$ \\
Uso ventilación mecánica & 55 & 25 & $<0,001$ & 15,6 & $7,7-31,5$ \\
Complicación en hospital & 125 & 34 & $<0,001$ & 10,4 & $5,0-21,3$ \\
\hline
\end{tabular}


modalidad forward y backward eliminando aquellas variables que no fueran incluidas en ninguno de los modelos. A cada variable retenida por el modelo se le asignó un puntaje mediante la amplificación de sus coeficientes de regresión, los cuales a su vez se agruparon en categorías de riesgo de acuerdo con la letalidad a 30 días.

Los modelos pronósticos diseñados en el análisis univariado y multivariado fueron comparados con los índices pronósticos descritos por Fine $^{7}$ y la Sociedad Británica de Tórax ${ }^{17}$ mediante curvas de receptor-operador (ROC), incluyendo sólo aquellos pacientes en los que se disponía de todas las variables clínicas y de laboratorio consideradas para el cálculo de los diferentes índices (391 individuos).

\section{Resultados}

En el período del estudio, se evaluaron 455 pacientes adultos inmunocompetentes hospitalizados por neumonía adquirida en la comunidad. Las características clínico-epidemiológicas, radiográficas y microbiológicas, la antibioticoterapia y evolución clínica han sido previamente descritas ${ }^{18,19}$.

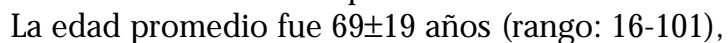
$55 \%$ eran varones, $76 \%$ tenía comorbilidad (especialmente cardiovascular, respiratoria y neurológica crónica) y, un tercio había recibido antibióticos antes del ingreso (especialmente ß-lactámicos, macrólidos y quinolonas). La estadía promedio en el hospital fue 9,9 $\pm 9,4$ días (rango: 1-54), un tercio presentó complicaciones médicas durante la evolución, 35 pacientes fallecieron en el hospital $(7,6 \%)$ y 46 pacientes fallecieron en el seguimiento a 30 días $(10,1 \%)$. En las Tablas 1 y 2, se describen las variables clínico-radiográficas asociadas a mayor riesgo de morir en el seguimiento a 30 días según el análisis univariado y se estratificaron las categorías de riesgo del índice clínico simple. Las tres categorías de riesgo están ampliamente representadas en la muestra poblacional y a medida que se asciende en la escala aumenta progresivamente el riesgo de muerte de los enfermos (Tabla 2).

El análisis multivariado mediante regresión logística permitió diseñar el índice pronóstico complejo, una segunda herramienta pronóstica que incluye las siguientes variables clínicas inde-
Tabla 2. Indice Pronóstico Simple derivado del análisis univariado de variables clínico-radiográficas asociadas a mortalidad en adultos hospitalizados por neumonía comunitaria

\begin{tabular}{|c|c|}
\hline Factores de riesgo & Puntaje \\
\hline Edad $>65$ años & 2 \\
\hline Comorbilidad (*) & 5 \\
\hline Sospecha de aspiración & 3 \\
\hline Duración síntomas $\leq 2$ días & 3 \\
\hline Ausencia de tos & 1 \\
\hline Compromiso de conciencia & 4 \\
\hline Ausencia de escalofríos & 3 \\
\hline $\mathrm{P}$ arterial $<90 / 60 \mathrm{mmHg}$ & 4 \\
\hline F respiratoria $>20 \times \mathrm{min}$ & 5 \\
\hline Temperatura $<37,5^{\circ} \mathrm{C}$ & 2 \\
\hline $\mathrm{SaO}_{2}<90 \%$ con $\mathrm{FiO}_{2}: 0,21$ & 2 \\
\hline NAC multilobar & 2 \\
\hline Rango & $0-36$ \\
\hline
\end{tabular}

(*) Comorbilidad: insuficiencia cardíaca congestiva, enfermedad pulmonar obstructiva crónica, diabetes mellitus, enfermedad cerebrovascular, neoplasia, insuficiencia renal crónica o enfermedad hepática crónica.

\begin{tabular}{|lcc|}
\hline Categorías de riesgo Pacientes & $\begin{array}{r}\text { Letalidad } \\
\text { a 30 días }\end{array}$ \\
\hline Categoría I (0 a 15 puntos) & 120 & $0,8 \%$ \\
Categoría II (16 a 20 puntos) & 110 & $5,4 \%$ \\
Categoría III (21 a 36 puntos) & 115 & $26,9 \%$ \\
\hline
\end{tabular}

pendientes no relacionadas: compromiso cualitativo de conciencia, presencia de comorbilidad, presión arterial sistólica inferior a $90 \mathrm{mmHg}$, temperatura axilar menor a $37,5^{\circ} \mathrm{C}$ y presencia de taquipnea, considerando dos rangos, entre 20 y 35 respiraciones por minuto y mayor a 35 respiraciones por minuto. A estas variables clínicas se les asignó un sistema de puntaje mediante la amplificación de sus coeficientes de regresión que osciló entre 0 y 14 puntos, estratificando a los pacientes en cuatro categorías de riesgo de acuerdo a la mortalidad a 30 días (Tabla 3). Se consideraron dos puntos de corte para la variable frecuencia respiratoria debido al valor pronóstico asignado a esta variable fisiológica en la mayoría de los 
estudios de factores pronósticos en neumonía comunitaria1-3,5-19 y la identificación de una categoría de enfermos con riesgo de muerte intermedio que presentaban valores de frecuencia

\section{Tabla 3. Indice Pronóstico Complejo derivado del análisis multivariado de variables clínico- radiográficas asociadas a mortalidad en pacientes hospitalizados por neumonía comunitaria}

\begin{tabular}{|c|c|c|}
\hline \multicolumn{2}{|l|}{ Factores de riesgo } & Puntaje \\
\hline \multicolumn{2}{|c|}{ Comorbilidad } & 3 \\
\hline \multicolumn{2}{|c|}{ Compromiso de conciencia } & 2 \\
\hline \multicolumn{2}{|c|}{ P arterial sistólica $<90 \mathrm{mmHg}$} & 3 \\
\hline \multicolumn{2}{|c|}{ F respiratoria $20-35 \times$ min } & 3 \\
\hline \multicolumn{2}{|c|}{ F respiratoria >35 x min } & 4 \\
\hline \multicolumn{2}{|l|}{ Temperatura $<37,5^{\circ} \mathrm{C}$} & 2 \\
\hline \multicolumn{2}{|l|}{ Rango } & $0-14$ \\
\hline Categorías de riesgo & Pacientes & $\begin{array}{l}\text { Letalidad } \\
\text { a } 30 \text { días }\end{array}$ \\
\hline Categoría I (0-5 puntos) & 116 & $0,9 \%$ \\
\hline Categoría II (6-7 puntos) & 123 & $4,9 \%$ \\
\hline Categoría III (8-9 puntos) & 106 & $14,2 \%$ \\
\hline Categoría IV ( $\geq 10$ puntos) & 59 & $35,6 \%$ \\
\hline
\end{tabular}

Tabla 4. C ategorías de riesgo y mortalidad a 30 días de los índices pronósticos de Fine ${ }^{7}$ y la Sociedad Británica de Tórax modificado ${ }^{17}$ en pacientes hospitalizados por neumonía comunitaria

\begin{tabular}{|lcc|}
\hline Indice de Fine & Pacientes & $\begin{array}{r}\text { Letalidad } \\
\text { a } 30 \text { días }\end{array}$ \\
\hline Clase I & 43 & $0 \%$ \\
Clase II & 59 & $0 \%$ \\
Clase III & 84 & $3,6 \%$ \\
Clase IV & 150 & $8,7 \%$ \\
Clase V & 117 & $25,6 \%$ \\
\hline Indice de BTS & Pacientes & Letalidad \\
(CURB-65) & & a 30 días \\
\hline Categoría I & 159 & $1,9 \%$ \\
Categoría II & 121 & $6,6 \%$ \\
Categoría III & 174 & $20,1 \%$ \\
\hline
\end{tabular}

respiratoria entre 20 y 35 respiraciones por minuto. Esto además, incrementa la sensibilidad y especificidad del modelo de regresión logística.

En las Tablas 4 y 5 se describe la estadía promedio en el hospital, tasa de complicaciones y mortalidad a 30 días de los pacientes hospitalizados por neumonía en las diferentes categoría de riesgo de los índices pronósticos de Fine y de la Sociedad Británica de Tórax. Los dos índices pronósticos clínicos diseñados en el presente estudio, que sólo incluyen información clínica y radiográfica, permiten estratificar a los enfermos en distintas categorías de riesgo de muerte y complicaciones en el hospital, similar al índice de gravedad descrito por Fine y el índice pronóstico de la Sociedad Británica de Tórax modificado (CURB-65). Así, los mayores puntajes se asocian

Tabla 5. Estadía en el hospital y tasa de complicaciones de los pacientes hospitalizados por neumonía comunitaria según las categorías de riesgo

\begin{tabular}{|c|c|c|}
\hline Indice Pronóstico & $\begin{array}{c}\text { Estadía } \\
\text { en hospital }\end{array}$ & Complicaciones \\
\hline \multicolumn{3}{|l|}{ Simple } \\
\hline Categoría I & $6,7 \pm 3,5 \mathrm{~d}$ & $14,9 \%$ \\
\hline Categoría II & $9,5 \pm 7,2 \mathrm{~d}$ & $25,4 \%$ \\
\hline Categoría III & $14,3 \pm 14,2 d^{*}$ & $44,3 \% *$ \\
\hline \multicolumn{3}{|l|}{ Complejo } \\
\hline Categoría I & $7,8 \pm 10,4 \mathrm{~d}$ & $14,5 \%$ \\
\hline Categonía II & $8,7 \pm 6,6 \mathrm{~d}$ & $29,3 \% *$ \\
\hline Categoría III & $11,5 \pm 9,0 d^{*}$ & $32,1 \% *$ \\
\hline Categoría IV & $14,4 \pm 12,5 \mathrm{~d}^{*}$ & $49,2 \% *$ \\
\hline \multicolumn{3}{|l|}{ Fine } \\
\hline Clase I & $5,8 \pm 3,4 \mathrm{~d}$ & $9,3 \%$ \\
\hline Clase II & $6,3 \pm 5,2 \mathrm{~d}$ & $11,9 \%$ \\
\hline Clase III & $10,2 \pm 13,0 \mathrm{~d}$ & $20,0 \%$ \\
\hline Clase IV & $9,2 \pm 6,1 \mathrm{~d}$ & $31,3 \% *$ \\
\hline Clase V & $13,8 \pm 11,0 \mathrm{~d}^{*}$ & $43,6 \% *$ \\
\hline \multicolumn{3}{|l|}{ BTS } \\
\hline Categoría I & $7,1 \pm 5,4 d$ & $13,8 \%$ \\
\hline Categoría II & $9,1 \pm 10,7 \mathrm{~d}$ & $27,9 \% *$ \\
\hline Categoría III & $12,9 \pm 10,2 d^{*}$ & $40,8 \% *$ \\
\hline
\end{tabular}

${ }_{p}<<0,01$ comparado con la categoría de menor riesgo. 
no sólo a una mayor mortalidad, sino que también a la aparición de complicaciones y una estadía más prolongada en el hospital.

En la Figura 1, aparece graficada la sensibilidad y especificidad de los dos índices pronósticos diseñados en este estudio, que consideraron información clínico-radiográfica exclusiva, siendo similares a los obtenidos por los índices pronósticos de Fine (PSI) y la BTS (CURB-65). El área bajo la curva de los índices pronósticos simple y complejo ( $A=0,83$ y 0,82 , respectivamente) fue semejante al área bajo la curva obtenida con los índices pronósticos descritos por Fine y la Sociedad Británica de Tórax ( $A=0,80$ y 0,75 , respectivamente).

\section{DisCUSIÓN}

En el presente estudio se ha logrado derivar dos índices pronósticos utilizando exclusivamente información clínica disponible en el medio ambulatorio a partir de una base de datos nacional de pacientes hospitalizados por neumonía comunitaria. La principal ventaja de ambos índices pronósticos es su simplicidad, incluyendo sólo cinco o doce parámetros clínico-radiográficos que suelen estar a disposición de los médicos de atención primaria, sin requerir de exámenes de laboratorio complementarios habitualmente disponibles sólo en el medio hospitalario. Además, constituye el primer instrumento pronóstico elaborado exclusivamente con información local, lo que facilitaría su aplicabilidad clínica en nuestro medio. Sin embargo, la principal limitación de nuestro modelo predictivo deriva del tamaño de la muestra examinada, considerando que los modelos predictivos extranjeros han contado con grandes tamaños muestrales para su derivación y validación $2,7,16,17$. De este modo, queda pendiente comprobar la utilidad clínica de ambos modelos predictivos en otras poblaciones de pacientes adultos inmunocompetentes atendidos en el medio ambulatorio de atención primaria (consultorios y servicios de urgencia) por neumonía comunitaria.

Parecido a lo comunicado en la literatura ${ }^{1-3,5-}$ 19 , en el análisis univariado las variables clínicoradiográficas asociadas a mortalidad elevada en el seguimiento a 30 días por neumonía comunitaria fueron: la edad avanzada ( $>65$ años), presencia de comorbilidad, enfermedad neurológica y hepática crónica, sospecha de aspiración, duración de los síntomas breve ( $\leq 2$ días), compromiso de concien-

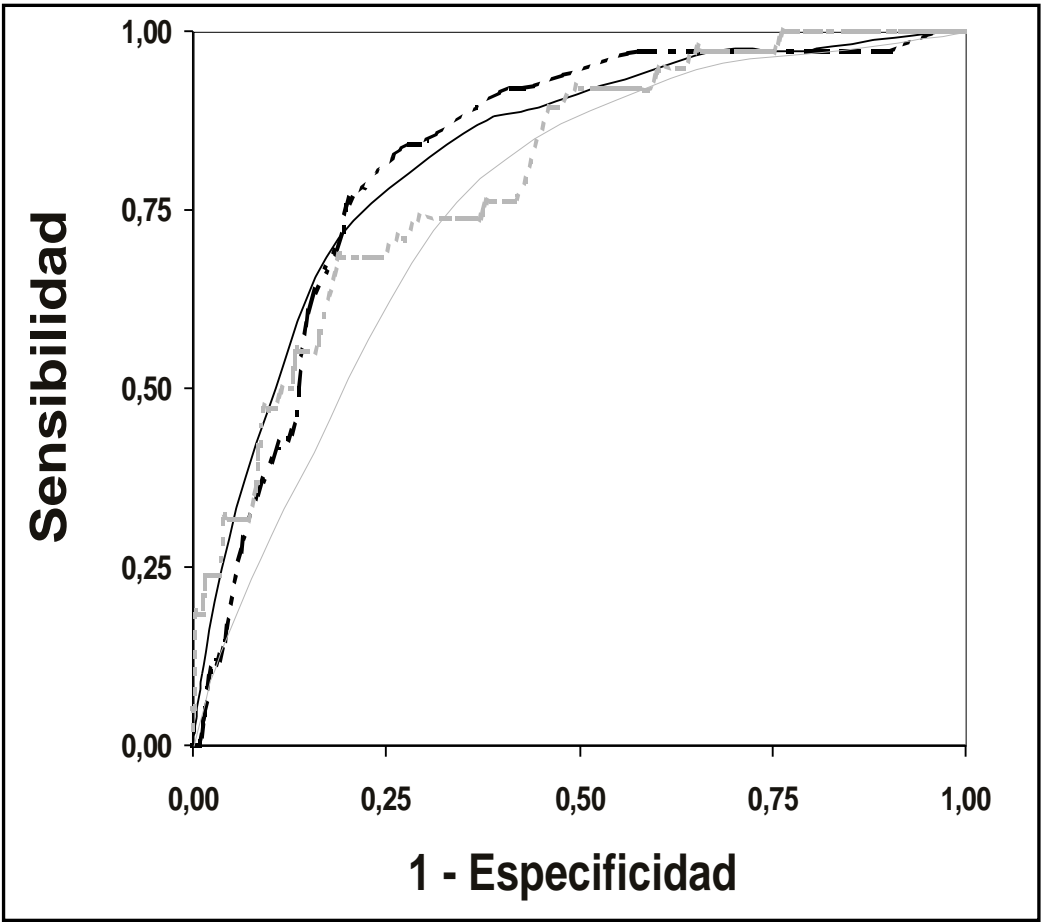

FiguRa 1. Curvas de receptor operador (ROC) para el índice clínico simple, el índice clínico complejo, el índice pronóstico de Fine (PSI) y el índice pronóstico de la Sociedad Británica de Tórax modificado (BTS). 
cia, ausencia de fiebre, tos y calofríos; hipotensión arterial, taquipnea, hipoxemia, el infiltrado radiográfico multilobar, la admisión a las Unidades de Cuidado Intermedio o Intensivo y el uso de ventilación mecánica. A partir del análisis univariado se ha diseñado el índice pronóstico clínico simple que incluye doce variables clínico-radiográficas y ha demostrado tener una elevada sensibilidad y especificidad en la detección de los pacientes de bajo riesgo de muerte y evolución favorable (categoría I), quienes pueden ser manejados en el medio ambulatorio, como se ha planteado con las categorías de bajo riesgo descritas por Fine y $\mathrm{col}^{7}$ (Tablas 2, 4 y 5). En el análisis multivariado permanecieron asociadas con mayor riesgo de morir en forma independiente las siguientes variables: la presencia de comorbilidad, hipotensión sistólica, compromiso de conciencia, taquipnea y ausencia de fiebre. Estos factores pronósticos medidos en la admisión al hospital también fueron descritos por Fine y cols, en su metaanálisis de factores pronósticos en neumonía comunitaria $^{6}$, que examinó alrededor de 122 estudios clínicos sobre el tema. Dentro de los factores de riesgo de evolución tórpida o muerte sugeridos por la Sociedad de Tórax Americana ${ }^{14}$ para considerar la hospitalización se incluyen los descritos en este estudio: edad $>65$ años, presencia de comorbilidad, compromiso de conciencia, hipotensión arterial, taquipnea, ausencia de fiebre y calofríos, hipoxemia y compromiso radiográfico multilobar. El índice pronóstico clínico complejo fue derivado del análisis multivariado, validando en nuestro medio la importancia de cinco variables clínicas en la detección de los pacientes con neumonía comunitaria de riesgo elevado: la presencia de comorbilidades específicas (insuficiencia cardíaca congestiva, enfermedad pulmonar obstructiva crónica, diabetes mellitus, enfermedad cerebrovascular, neoplasia activa, insuficiencia renal crónica y enfermedad hepática crónica), el estado mental alterado, hipotensión arterial, taquipnea y ausencia de fiebre. De acuerdo al puntaje obtenido, los pacientes fueron estratificados en cuatro categorías de riesgo, lo que permitiría definir el pronóstico, orientar el lugar de manejo y el tratamiento de los pacientes adultos inmunocompetentes atendidos por neumonía comunitaria en el medio ambulatorio.
Dentro de las limitaciones del presente estudio, es probable que las distintas comorbilidades confieran un riesgo de muerte o complicaciones diferentes a los pacientes con neumonía comunitaria. Sin embargo, considerando el tamaño muestral limitado y los distintos criterios empleados en la definición de las enfermedades crónicas, los estudios de factores pronósticos en el medio nacional e internacional (JAMA 1996; 275: 134-41) han optado por utilizar definiciones generales de las enfermedades crónicas y han evitado su categorización por gravedad.

En el presente estudio se ha demostrado que los dos índices pronósticos clínicos, tal como ocurre con el índice de gravedad de Fine y de la Sociedad Británica de Tórax, son aplicables en nuestra población, con niveles de sensibilidad y especificidad semejantes a los índices pronósticos extranjeros. Sin embargo, el mayor aporte que realiza nuestro modelo sobre aquellos previamente descritos en la literatura extranjera, es su simplicidad y fácil aplicabilidad en el contexto de la atención primaria (consultorios y servicios de urgencia); donde puede ayudar a discriminar entre un grupo de pacientes de bajo riesgo (categoría I), que pueden ser tratados eficazmente en el medio ambulatorio; otro grupo que puede requerir una hospitalización abreviada o vigilancia cercana en el sistema ambulatorio (categoría II) y, una tercera categoría de riesgo elevado que requeriría hospitalización ya sea en sala de cuidados generales 0 en una unidad de cuidados especiales (categorías III y IV, respectivamente). Esta distinción puede hacerse de manera rápida, sin la necesidad de exámenes de laboratorio, considerando que el puntaje que se asigne al paciente no sólo nos informa sobre su probabilidad de fallecer, sino que además de su riesgo de presentar complicaciones en la evolución o necesidad de hospitalización prolongada.

Sin embargo, las decisiones clínicas se deben adoptar mediante una valoración global del contexto médico y social en el cual la enfermedad acontece; así, los índices pronósticos derivados de modelos matemáticos deberían ser empleados como elementos de orientación y nunca deberían reemplazar el juicio clínico. De este modo, los índices clínicos propuestos y el índice de gravedad de Fine no consideran factores sociales y de 
adherencia al tratamiento en la decisión de hospitalización, variables que deben ser consideradas por el clínico.

En resumen, en el presente estudio se describen las características clínico-epidemiológicas y factores pronósticos asociados a mortalidad elevada en los adultos inmunocompetentes hospitalizados por neumonía adquirida en la comunidad en nuestro medio, y se han construido dos herramientas clínicas que pueden ser utilizadas en el medio ambulatorio para la evaluación de la gravedad y decidir el lugar de manejo. La deriva-

\section{REFERENCIAS}

1. Garibaldi RA. Epidemiology of community-acquired respiratory tract infections in adults: incidence, etiology and impact. Am J Med 1985; 78: 32-7.

2. Harrison BDW, Farr BM, Pugh S, Selkon JB. Community-acquired pneumonia in adults in British hospitals in 1982-1983: a survey of aetiology, mortality, prognostic factors and outcome. Q J Med 1987; 62: 195-220.

3. BaRTLETT JG, Mundy LM. Community-acquired pneumonia. N Engl J Med 1995; 333: 1618-24.

4. Programa de Infecciones Respiratorias Agudas (IRA), Departamento de Estadísticas e Información de Salud, Ministerio de Salud de Chile, 2001.

5. Neill AM, Martin IR, Weir R, Anderson R, ChereshsKY A, EptON MJ ET AL. Community acquired pneumonia: aetiology and usefulness of severity criteria on admission. Thorax 1996; 51: 1010-6.

6. Fine MJ, Smith MA, Carson CA, Mutha SS, Sankey SS, WeISSFeld LA ET AL. Prognosis and outcomes of patients with community-acquired pneumonia. A meta-analysis. JAMA 1996; 275: 134-41.

7. Fine MJ, Auble TE, Yealy DM, Hanusa BH, WeissFELD LA, Singer DE ET AL. A prediction rule to identify low-risk patients with community-acquired pneumonia. N Engl J Med 1997; 336: 243-50.

8. Leroy O, Santre C, Beuscart C. A 5 years study of severe community-acquired pneumonia with emphasis on prognosis in patients admitted to an ICU. Intensive Care Med 1995; 21: 24-31.

9. Ruiz M, Ewig S, Torres A, Arancibia F, Marco F, MENSA J ET AL. Severe community-acquired pneu- ción de índices pronósticos de una cohorte de pacientes hospitalizados por neumonía comunitaria no necesariamente será de utilidad en la evaluación de pacientes ambulatorios con bajo riesgo de muerte. Queda como tarea pendiente realizar la validación de ambos índices pronósticos clínicos en otras poblaciones de pacientes ambulatorios atendidos en centros de atención primaria (consultorios y servicios de urgencia) por infecciones del tracto respiratorio, con el propósito de racionalizar el manejo de la neumonía comunitaria en el país.

monia: risk factors and follow-up epidemiology. Am J Respir Crit Care Med 1999; 160: 923-9.

10. Riquelme R, Torres A, El-Ebiary M, Puig De La Belacasa J, Estruch R, Mensa J et al. Communityacquired pneumonia in the elderly. A multivariate analysis of risk and prognostic factors. Am J Respir Crit Care Med 1996; 154: 1450-5.

11. Re山o J, Rodríguez R, Jubert P, Alvarez B. Severe community-acquired pneumonia in the elderly: epidemiology and prognosis. Study Group for Severe Community-Acquired Pneumonia. Clin Infect Dis 1996; 23: 723-8.

12. Ewig S, Kieinfeld T, Bauer T, Seifert $K$, SchäFer $H$, GÖKE N. Comparative validation of prognostic rules for community-acquired pneumonia in an elderly population. Eur Respir J 1999; 14: 370-5.

13. Houston MS, SilverStein MD, Suman VJ. Risk factors for 30 days mortality in elderly patients with lower respiratory tract infection. Community-based study. Arch Intern Med 1997; 157: 2190-5.

14. Niederman MS, Mandell LA, Anzueto A, Bass JB, Broughton WA, CAmpBell GD et al. Guidelines for the management of adults with communityacquired pneumonia. Diagnosis, assessment of severity, antimicrobial therapy and prevention. Am J Respir Crit Care Med 2001; 163: 1730-54.

15. BRitish Thoracic Society Standards of Care CommitTEE. British Thoracic Society guidelines for the management of community-acquired pneumonia in adults. Thorax 2001; 56 (suppl IV): 1-64.

16. LM WS, LEWIS S, MacFARLANE JT. Severity prediction rules in community acquired pneumonia: a validation study. Thorax 2000; 55: 219-23. 
17. Lim WS, Van Der Eerden MM, Laing R, Boersma WG, Karalus N, Town GI et al. Defining community acquired pneumonia severity on presentation to hospital: an international derivation and validation study. Thorax 2003; 58: 377-82.

18. Saldías F, Mardonez JM, Marchesse M, Viviani $P$, Farías G, Díaz A. Neumonía adquirida en la comunidad en el adulto hospitalizado. Cuadro clínico y factores pronósticos. Rev Méd Chile 2002; 130: 1373-82.

19. Saldías F, O'brien A, Gederlini A, Farías G, díaz A. Neumonía adquirida en la comunidad en el anciano inmunocompetente que requiere hospi- talización. Cuadro clínico, factores pronósticos y tratamiento. Arch Bronconeumol 2003; 39: 33340.

20. Fang GD, Fine M, Orloff J, Arisumi D, Yu VL, KAPOOR W ET AL. New emerging etiologies for community-acquired pneumonia with implications for therapy. A prospective multicenter study of 359 cases. Medicine (Baltimore) 1990; 69: 307-16.

21. Ruiz M, Ewig S, Marcos MA, Martínez JA, Arancibia F, MENSA J ET AL. Etiology of community-acquired pneumonia: impact of age, comorbidity and severity. Am J Respir Crit Care Med 1999; 160: 397-405. 